\title{
Molecular Diversity of Drug Resistant Mycobacterium Tuberculosis Strains in Western Turkey
}

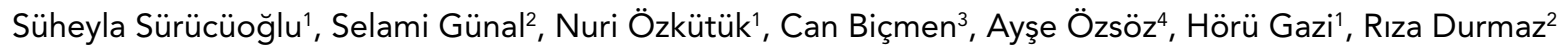 \\ ${ }^{1}$ Department of Medical Microbiology, Faculty of Medicine, Celal Bayar University, Manisa, Turkey \\ 2Department of Medical Microbiology, Faculty of Medicine, Inönü University, Malatya, Turkey \\ ${ }^{3}$ Department of Medical Microbiology, Dr. Suat Seren Chest Diseases and Surgery Training and Research Hospital, Izmir, Turkey \\ ${ }^{4}$ Department of Tuberculosis and Chest Diseases, Dr. Suat Seren Chest Diseases and Surgery Training and Research Hospital, Izmir, Turkey
}

\section{ABSTRACT}

Objective: The aim of this study was to investigate the molecular diversity and clonal relationship of drug resistant Mycobacterium tuberculosis strains isolated in Western Turkey.

Materials and Methods: A total of 87 strains isolated between 2006 and 2009, eight of which were rifampicin monoresistant and 79 were multidrug resistant, were analyzed with IS6110 RFLP and spoligotyping methods.

Results: The results of spoligotyping showed that 7\% of the strains were orphans, and $8 \%$ were undefined for family in the SpolDB4 database. Major families of the strains were LAM (38\%), T (35\%), Haarlem (7\%), Beijing (2\%), S (2\%) and U (1\%) families. The clustering rate by spoligotyping was calculated as $75 \%$. The most predominant SIT cluster was SIT41 (29\%). According to the results of IS6110 RFLP, 71 different patterns of IS6110 were observed. Low copy number was found in $26 \%$ of the strains. When the results of two methods were combined, the final clustering rate was calculated as $26 \%$.

Conclusions: The genotypical distribution of drug resistant tuberculosis isolates in our region indicates genetic diversity and the clustering rate was found low in our region. However, more comprehensive and long-term molecular epidemiological studies are needed to control the drug resistant strains.

Key Words: Mycobacterium tuberculosis, drug resistance, genotyping, epidemiology

Received: 20.06.2011 Accepted: 14.02.2012

\section{Introduction}

Drug resistant tuberculosis (TB) is a major public health problem due to the difficulty in treating the disease and increased risk of spreading drug resistant isolates in the community $(1,2)$. According to the World Health Organization (WHO) Report in 2008 (WHO/HTM/TB/2009.426), almost 30.000 multidrug resistant tuberculosis (MDR-TB) cases were reported worldwide (3). Drug resistant TB is also a major problem in Turkey. Turkey's population is 74.877 .000 and the incidence of TB is 25 per 100.000 . According to the WHO report, the number of patients diagnosed with MDR-TB in Turkey was 240 in 2008 (WHO/HTM/TB/2009.426). However, its incidence and prevalence might be higher due to under-reporting to the health authorities. Turkey is a geographic bridge between Europe and Asia and has received a considerable number of immigrants from Eastern Europe and Asian countries. Furthermore, local population movements from rural to urban areas and between cities are high. The studies on molecular epidemiology of drug resistant TB could allow tracking of the transfer path of the resistant strains $(4,5)$. In Turkey, the molecular analysis of Mycobacterium tuberculosis strains has been performed only in regional studies. There are also a small number of studies on molecular epidemiology of drug resistant TB in Turkey (68). In this study, we aimed to determine the genetic diversity and clonal relationship of drug resistant strains isolated from patients living in our region, Western Turkey, through IS6110 RFLP and spoligotyping methods.

\section{Material and Methods}

\section{M. tuberculosis strains and Patients}

A total of 87 Mycobacterium tuberculosis strains, 79 of which were multidrug resistant (MDR) and eight were rifampicin monoresistant that were isolated from respiratory tract samples taken in Izmir Dr. Suat Seren Chest Diseases and Surgery Training and Research Hospital between 2006 and 2009 , were included in the study. Strains were identified with a commercial line probe assay (Hain Lifescience $\mathrm{GmbH}$ ). Drug resistance tests for the first generation drugs, rifampicin (R) and streptomycin (S), isoniazid (I) and ethambutol (E), was performed by the modified $1 \%$ proportion method in the BACTEC 460TB system (9). The strains were stored in Löwenstein Jensen medium at $+4^{\circ} \mathrm{C}$ for further genetic examination. 


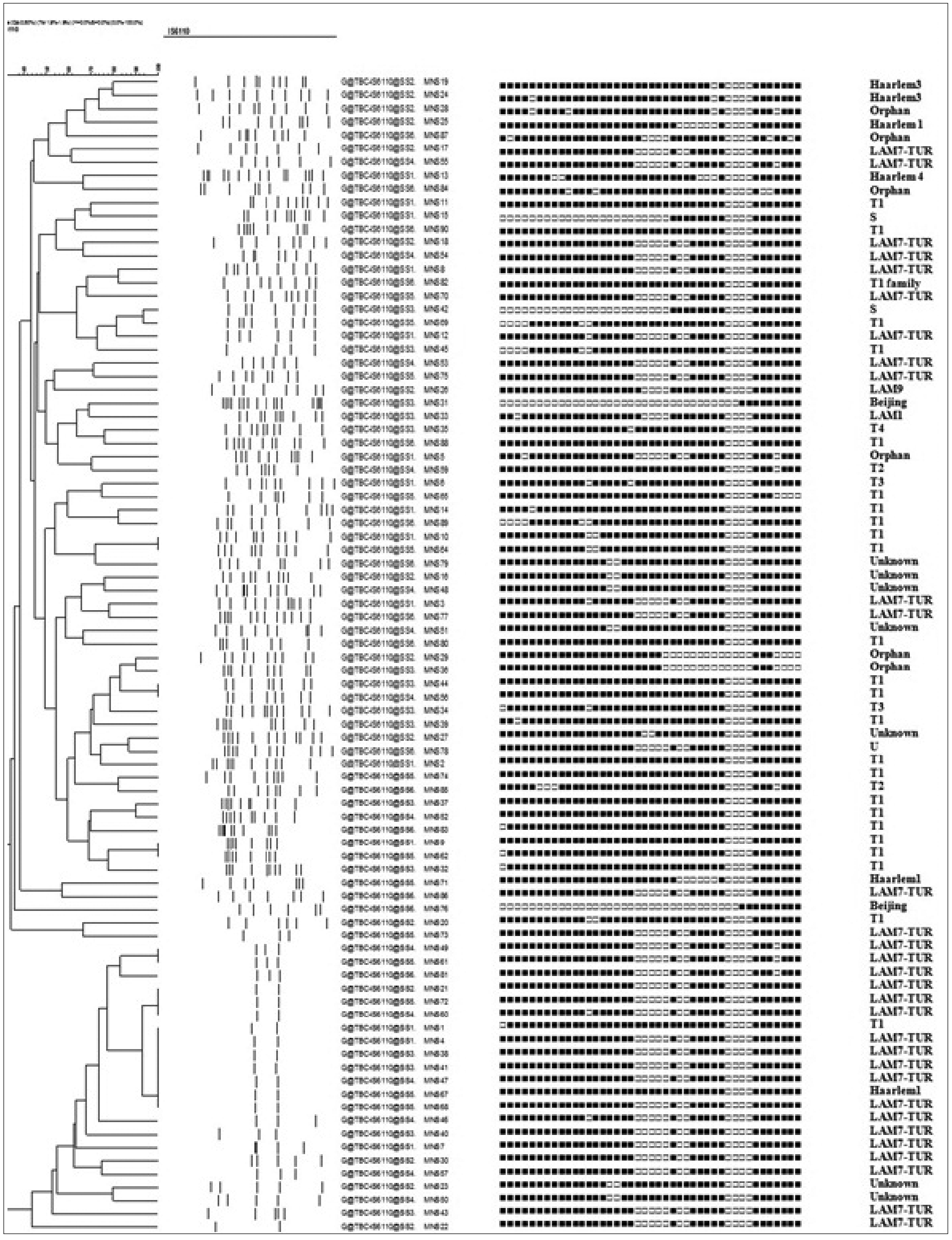

Figure 1. Dendrogram based on the IS6110 RFLP results. Spoligotypes and the families are shown on the right side of the figure 
Only the first strain among the repeated strains isolated from the same patient was selected for genotyping in the study.

Patients' histories of therapy were reviewed from patient records and the drug resistance in patients who had not received treatment before, or who received treatment for less than a month, was defined as "drug-resistance in new cases (used to be initial resistance)". Drug-resistance in patients who received treatment for at least one month was defined as "drug-resistance in previously treated patients (used to be acquired resistance)" (10). The study was approved by the University's ethic committee.

\section{Genotyping Methods}

The strains were genotyped by RFLP DNA fingerprinting method and spoligotyping in Molecular Microbiology Laboratory of Molecular Microbiology Laboratory of Inonu University Medical Faculty in Malatya. Strains were classified in a cluster if they showed: (a) 100\% identical IS6110 RFLP patterns having six or more hybridizing bands, (b) IS6110 RFLP patterns having six or more bands that differed by a single band (similar patterns) but identical spoligotype patterns, (c) $100 \%$ identical IS6110 RFLP fingerprint patterns with less than six bands, and identical spoligotype pattern (8). Standard spoligotyping was performed with the Dra and Drb primers with Dra biotinylated in 5', as described previously by Kamerbeek (11). Spoligotypes in binary format were entered in an Excel spreadsheet and compared to the SpoIDB4 database, which was the international spoligotyping database of the Pasteur Institute of Guadeloupe, (http://www.pasteur-guadeloupe.fr:8081/SITVITDemo). IS6110 RFLP was performed using standardized methodology described previously by van Embden et al. (12). The results were analyzed with Taxotron; a pair-wise distance matrix was built by using the Dice Index.

\section{Results}

\section{Patients and the drug-resistance patterns of M. tuberculosis strains}

Sixty-six patients (76\%) were males and 21 patients (24\%) were females. Fifteen of the patients (17\%) had just received the diagnosis and, therefore, history of treatment was not available. The drug-resistance in these patients was considered as initial resistance. The drug resistance in bacteria isolated from 72 patients (83\%) who had histories of treatment was considered as acquired drug resistance. The most frequent drug-resistance phenotypes were I+R (26.44\%) and resistance to four drugs concurrently (26.44\%). Other resistance phenotypes were I+R+E (24.14\%), S+I+R (13.79\%), and R monoresistance (9.19\%).

\section{The Results of Spoligotyping}

As a result of spoligotyping, six strains (7\%) were identified as orphans and the families of seven strains $(8 \%)$ could not be identified on SpoIDB4 database. The major families that the remaining 74 strains belonged to were Latin American and Mediterranean (LAM) (33 strains, 38\%), T (30 strains, 35\%), Haarlem (6 strains, 7\%), Beijing (2 strains, 2\%), S (2 strains, 2\%) and $U(1$ strain, $1 \%)$. When spoligotype patterns of $M$. tuberculosis strains were explored using SpolDB4 database, it was observed that strains had shared 27 different international common spoligotye patterns (SITs-Shared international spoligotypes) while 65 strains shared 11 SIT patterns (2-23 strains). Through spoligotyping the clustering rate was calculated as $75 \%$. The most predominant (29\%) SIT cluster was SIT41 (LAM7-TUR Family) where 23 strains were clustered, followed by SIT53 (T1 family), SIT2067 (unknown), SIT7 (T1 family), SIT367 (LAM7-TUR family), SIT1261 (LAM7-TUR family), SIT131 (T1 family), SIT284 (T1 family), SIT1 (Beijing family), SIT4 (S family) and SIT47 (Haarlem 1 family). Data on specific spoligotype patterns of 81 strains and their SITs are given in Table 1.

\section{Results of IS6110 RFLP}

According to the results of IS6110 RFLP, 71 different IS6110 RFLP patterns were observed. The number of copies among these patterns was between 2-16. Twenty-three of the strains $(26 \%)$ had a low number of copies $(<6)$. It was detected that 23 of the 87 strains comprised seven clusters (clustering rate $26 \%)$. The size of the clusters ranged from 2 to 10 strains and the largest cluster with 10 strains (C7) was observed among the strains having two copies of IS6110 RFLP.

\section{Combination of the Results of IS6110 RFLP and Spoligotyping}

The results of two genotyping methods were assessed together to differentiate those strains that had less than six copies by the IS6110 RFLP method. As a result of spoligotyping, three of 10 strains in cluster $\mathrm{C} 7$ were excluded from the cluster. Finally a total of seven clusters with 73 different genotypes were seen and the clustering rate was calculated as $23 \%$. Two strains which were found to be identical by IS6110 RFLP turned out to be different by spoligotyping. Spoligotype of the first patient was determined to be SIT4 and of the second as SIT41. Since there was no history of contact between two patients, the strains were not included in the same cluster. Figure 1 shows a dendrogram based on the results of IS6110 RFLP and spoligotype patterns.

When the drug resistance features of 20 strains in seven clusters identified by the combination of IS6110 RFLP and spoligotyping were examined, it was observed that three strains had an initial resistance, whereas 17 strains had acquired drug resistance. When these cases were investigated, a history of household contact was detected in only one patient (cluster C7), whereas no source could be found in the other patients. The cluster $\mathrm{C7}$ with seven members, the most crowded cluster, has a SIT41 spoligotype which is from the LAM7-TUR family. The drug resistance features and SIT patterns of 20 clustered strains are shown in Table 2.

In the study, two strains in the Beijing family were found to be different by IS6110 RFLP. One of these two strains belonged to a female patient of Azerbaijan origin and carried $\mathrm{R}+\mathrm{I}$ drug resistance. The second strain belonged to a 40-year old female patient living in Izmir and lacked any history of contact and carried $\mathrm{R}$ monoresistance.

\section{Discussion}

Researches on molecular epidemiology of drug resistant tuberculosis provide significant contributions to TB control 
Table 1. The spoligotyping results of $M$. tuberculosis strains*

\begin{tabular}{|c|c|c|c|c|c|}
\hline Spoligotypes & Octal Code & SITs & Family & $\begin{array}{c}\text { No of the } \\
\text { strains }\end{array}$ & SITs \% \\
\hline 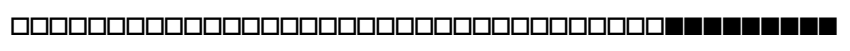 & 000000000003771 & 1 & Beijing & 2 & 3 \\
\hline 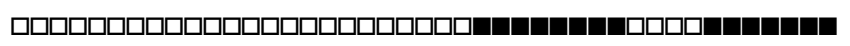 & 000000007760771 & 4 & $\mathrm{~S}$ & 2 & 3 \\
\hline 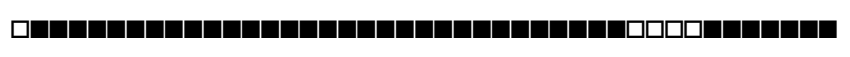 & 377777777760771 & 7 & $\mathrm{~T} 1$ & 4 & 5 \\
\hline 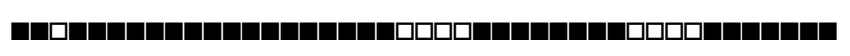 & 677777607760771 & 20 & LAM1 & 1 & 1 \\
\hline m & 777777377760771 & 40 & T4 & 1 & 1 \\
\hline m & 777777404760771 & 41 & LAM7-TUR & 23 & 29 \\
\hline $\boldsymbol{\square}$ & 777777607760771 & 42 & LAM9 & 1 & 1 \\
\hline 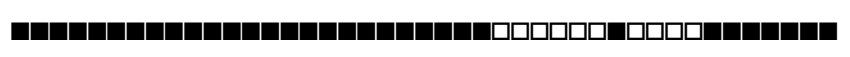 & 777777774020771 & 47 & $\mathrm{H} 1$ & 2 & 3 \\
\hline 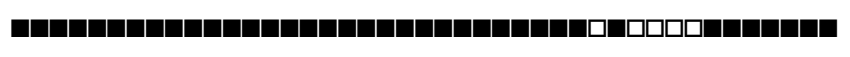 & 777777777720771 & 50 & $\mathrm{H} 3$ & 1 & 1 \\
\hline 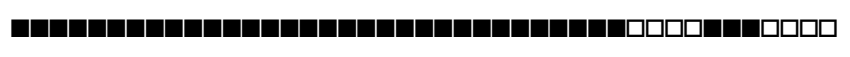 & 777777777760700 & 51 & $\mathrm{~T} 1$ & 1 & 1 \\
\hline 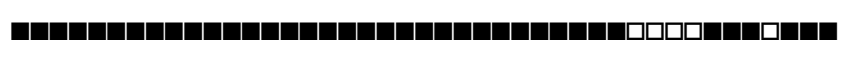 & 777777777760731 & 52 & $\mathrm{~T} 2$ & 1 & 1 \\
\hline 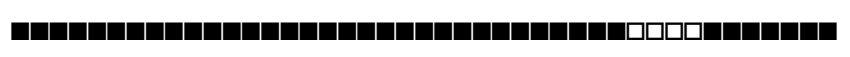 & 777777777760771 & 53 & $\mathrm{~T} 1$ & 12 & 15 \\
\hline Iप्रण & 757777777720771 & 99 & $\mathrm{H} 3$ & 1 & 1 \\
\hline प्रपा & 777717777760771 & 131 & $\mathrm{~T} 1$ & 3 & 4 \\
\hline I0त्र & 757400004020771 & 143 & $\mathrm{H} 1$ & 1 & 1 \\
\hline 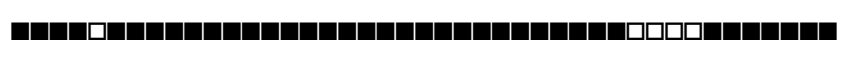 & 757777777760771 & 154 & $\mathrm{~T} 1$ & 1 & 1 \\
\hline 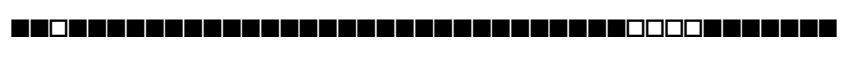 & 677777777760771 & 196 & $\mathrm{~T} 1$ & 1 & 1 \\
\hline Iप्रण & 774777777420771 & 262 & $\mathrm{H} 4$ & 1 & 1 \\
\hline 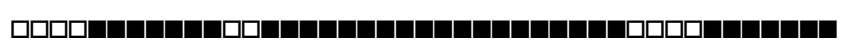 & 037637777760771 & 284 & $\mathrm{~T} 1$ & 3 & 4 \\
\hline סחص & 777737404760771 & 367 & LAM7-TUR & 4 & 5 \\
\hline טח & 777737377760771 & 442 & T3 & 1 & 1 \\
\hline סחص & 777777770000771 & 602 & $U$ & 1 & 1 \\
\hline 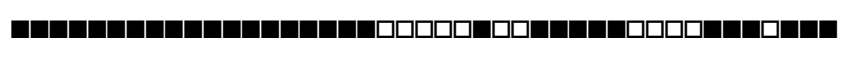 & 777777404760731 & 1261 & LAM7-TUR & 4 & 5 \\
\hline 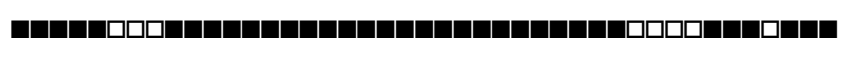 & 761777777760731 & 1622 & $\mathrm{~T} 2$ & 1 & 1 \\
\hline 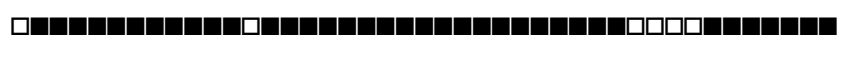 & 377737777760771 & 1834 & T3 & 1 & 1 \\
\hline 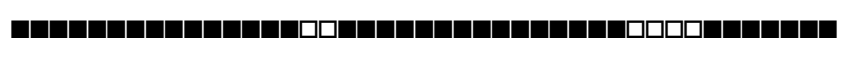 & 777771777760771 & 2067 & Unknown & 6 & 8 \\
\hline 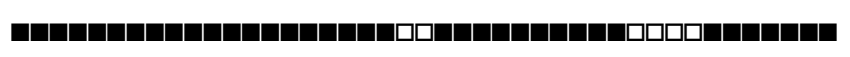 & 777777637760771 & 2070 & Unknown & 1 & 1 \\
\hline
\end{tabular}

programs by giving information about the transmission of resistant strains in the study area. In spoligotyping studies carried out in our country, the clustering rate ranges between $67-85 \%(6,8,13-16)$. This rate is unrealistically high. When the results are repeated by the IS6110 RFLP and/or MIRU methods, the rates of clustering are markedly reduced. In this study, the clustering rate was calculated as $75 \%$ by spoligotyping and dropped to $23 \%$ as a result of IS6110 RFLP analysis.

The most frequent spoligotype (29\%) in our region is SIT41 from the LAM7-TUR family. SIT41, phylogeographically, is a genotype that belongs to Asia Minor and is prevalent in our country (8). The results of spoligotyping performed by Durmaz et al. (8) for drug-resistant strains obtained from different areas of our country showed that SIT41, with a rate of $23 \%$, is the most frequent genotype. In the spoligotyping studies carried by Kisa et al. (15) in Ankara (Capital city, Central Anatolia) and by Aktaş et al. (13) in Zonguldak (Northern Turkey) SIT53 was reported to be the most frequent genotype (32\% and $22 \%$, respectively), followed by SIT41. However, in both studies, besides strains resistant to drugs, sensitive strains were also included. In our study, SIT53 belonging to the T super family was the second most frequent genotype (15\%). SIT53 demonstrates an equal prevalence worldwide as well as in our country (8). Other families, namely Haarlem (7\%), Beijing (2\%) and S (\%2) are less frequent in our region. The Haarlem family is prevalent in our country as well as other European countries $(8,16)$. While the Haarlem family represents $8 \%$ of cases in Europe, the frequency of Beijing family is $4 \%$ (17). In Europe, however, the epidemiologic and molecular analyses performed on 2.494 MDR-TB cases between 2003-2007 revealed that Beijing genotype was the most frequent genotype, with $55 \%$ (18). Since some genotypes such as Beijing 
Table 2. Drug resistance and SITs patterns of $20 \mathrm{M}$. tuberculosis strains clustered by two genotyping methods

\begin{tabular}{|lcccc|}
\hline Cluster & Number of the strains & Drug resistance patterns & Resistance & \multicolumn{1}{c|}{ SITs } \\
\hline C1 & 2 & $R$ & Acquired & SIT131 \\
C3 & 1 & $\mathrm{~S}+\mathrm{I}+\mathrm{R}+\mathrm{E}$ & Initial & SIT53 \\
& 1 & $\mathrm{~S}+\mathrm{I}+\mathrm{R}+\mathrm{E}$ & Acquired & \\
C4 & 1 & $\mathrm{~S}+\mathrm{I}+\mathrm{R}+\mathrm{E}$ & Acquired & SIT53 \\
C5 & 1 & $\mathrm{~S}+\mathrm{I}+\mathrm{R}$ & Initial & \\
C6 & 2 & $\mathrm{I}+\mathrm{R}$ & Acquired & SIT53 \\
& 2 & $\mathrm{I}+\mathrm{R}+\mathrm{E}$ & Acquired & Unknown (SIT2067) \\
C7* & 2 & $\mathrm{~S}+\mathrm{I}+\mathrm{R}$ & Acquired Acquired & SIT1261 \\
& 1 & $\mathrm{~S}+\mathrm{I}+\mathrm{R}+\mathrm{E}$ & & \\
& 3 & $\mathrm{~S}+\mathrm{I}+\mathrm{R}+\mathrm{E}$ & Acquired & SIT41 \\
& 1 & $\mathrm{I}+\mathrm{R}+\mathrm{E}$ & Initial* & \\
& 2 & $\mathrm{I}+\mathrm{R}$ & Acquired Acquired & \\
\hline *The case had a history of household contact, the strain was isolated in 2006 & $\mathrm{R}$ & & \\
\hline
\end{tabular}

and Haarlem are frequently found in drug-resistant strains and it takes a relatively long time for smears to be positive, they possess certain clinical and epidemiologic characteristics such as prolonged transmission (18). Beijing family is encountered especially in Asian and Eastern European countries. However, immigrations to our country from such regions may result in an increase in the frequency of this genotype. Two of the strains examined in this study belonged to Beijing family. One of the subjects was a patient who emigrated from Azerbaijan and the strain was found to have $\mathrm{R}+\mathrm{I}$ resistance. No epidemiologic link could be detected in the second subject. In a study carried out in Istanbul, only $1.13 \%$ of 4069 strains were identified to have Beijing genotype (16). The higher rate (2\%) found in the present study may have resulted from working with MDR strains. The CAS (Central Asia) and EAI (East African Indian) families which are highly prevalent in Asian countries besides Beijing are never identified in our region.

In the present study, we found that $26 \%$ of the strains had a low copy count. A research carried out by Bicmen et al. (19) in our region in 2007 reported the frequency of strains with a low copy count as $25 \%$ and clustering rate as $18 \%$. Another study conducted by Cavusoglu et al. (7) in 2004 with strains resistant to rifampicin, found the frequency of strains with low copy count and the clustering rate as $27 \%$ and $35 \%$, respectively. It is known that the clustering rate is much higher in resistant strains, a fact attributed to the infectivity period of resistant strains being longer. In the Durmaz et al study (8), the clustering rate in MDR strains was $40 \%$, in contrast to $21 \%$ in non-MDR strains. In a research study carried out in Europe regarding molecular surveillance on MDR-TB cases, molecular and epidemiologic relationships were detected in $39 \%$ of cases (4). In Estonia, which is one of the countries where MDR-TB cases are most frequently seen, the clustering rate was found as $49 \%$. The authors determined that $88 \%$ of MDR strains, $67 \%$ of strains resistant to at least one drug and only $12.2 \%$ of sensitive strains, belonged to one family (5). However, in contrast to these data, there are studies which reported that strains resistant to drugs had limited transmission (20).

\section{Conclusion}

The clustering rate of MDR-TB isolates in our region is $23 \%$. The most prevalent genotype is SIT41 from the LAM7TUR family, which is widespread in our country. The Beijing genotype with high drug resistance and transmission feature has been defined, albeit it has reduced frequency. In order to control these strains, epidemiologic research should continue. In our region, the clustering rate in MDR strains was found low. However, there is a need for more comprehensive and long-term molecular epidemiologic research, including a wider range of strains.

\section{Conflict of Interest}

No conflict of interest was declared by the authors.

\section{References}

1. Dye C, Espinal MA, Watt CJ, Mbiaga C, Williams BG. Worldwide incidence of multidrug-resistant tuberculosis. J Infect Dis 2002;15:1197-202. [CrossRef]

2. Mathema B, Kurepina NE, Bifani PJ, Kreiswirth BN. Molecular epidemiology of tuberculosis: Current insights. Clin Microbiol Rev 2006;19:658-85. [CrossRef]

3. Global tuberculosis control: A short update to the 2009 report. World Health Organization (WHO/HTM/TB/2009.426), http:// www.who.int/tb/publications/global_report/2009/update/en/

4. Devaux I, Kremer K, Heersma H, van Soolingen D. Clusters of multidrug-resistant Mycobacterium tuberculosis cases, Europe. Emerg Infect Dis 2009;15:1052-60. [CrossRef]

5. Krüüner A, Hoffner SE, Sillastu H, Danilovits M, Levina K, Svenson $\mathrm{SB}$, et al. Spread of drug-resistant pulmonary tuberculosis in Estonia. J Clin Microbiol 2001;39:3339-45. [CrossRef]

6. Durmaz R, Zozio T, Gunal S, Allix C, Fauville-Dufaux M, Rastogi N. Population based molecular epidemiology study of tuberculosis in Malatya, Turkey. J Clin Microbiol 2007;45:4027-35. [CrossRef] 
7. Cavusoglu C, Durmaz R, Bilgic A, Gunal S. Genotyping of rifampin-resistant Mycobacterium tuberculosis isolates from western Turkey. Ann Saudi Med 2004;24:102-5.

8. Durmaz R, Zozio T, Gunal S, Yaman A, Cavusoglu C, Guney C, et al. Genetic diversity and major spoligotype families of drug resistant Mycobacterium tuberculosis clinical isolates from different regions of Turkey. Infect Genet Evol 2007;7:513-9. [CrossRef]

9. Sıddıqi SH. Drug susceptibility testing. In: BACTEC TB System product and procedure manual (Revision E). Maryland: Becton Dickinson 1996;IV:1-IV.23.

10. TheWHO/IUATLD global project on antituberculosis drug resistance surveillance. Anti-tuberculosis drug resistance in the world, report no:4. http://whqlibdoc.who.int/hq/2008/WHO_HTM_ TB_2008.394_eng.pdf

11. Kamereek J, Schouls L, Kolk A, van Agterveld M, van Soolingen $D$, Kuijper $S$, et al. Simultaneous detection and strain differentiation of Mycobacterium tuberculosis for diagnosis and epidemiology. J Clin Microbiol 1997;35:907-14.

12. van Embden JDA, Cave MD, Crawford JT, Dale JV, Eisenach KD, et al. Strain identification of Mycobacterium tuberculosis by DNA fingerprinting: Recommendations for a standardized methodology. J Clin Microbiol 1993;31:406-9.

13. Aktas E, Zozio T, Comert FB, Kulah C, Aydin O, Rastogi N, et al. $A$ first insight into the gentic diversity and population structure of
Mycobacterium tuberculosis in Zonguldak, Turkey. Clin Microbiol Infect 2008;14:55-9. [CrossRef]

14. Gencer B, Shinnick TM. Molecular genotyping of Mycobacterium tuberculosis isolates from Turkey. Am J Infect Dis 2005;1:5-11. [CrossRef]

15. Kisa O, Albay A, Baylan O, Tozkoparan E, Acikel CH, Doganci L. Genetic diversity of Mycobacterium tuberculosis isolates at the Military Medical Academy in Ankara, Turkey. Res in Microbiol 2007;158:318-23. [CrossRef]

16. Koksalan OK, Kilicaslan Z, Zanlier G, Guzel R, Seber E. Prevalance of Beijing genotype Mycobacterium tuberculosis strains in Istanbul. Int J Tuberc Lung Dis 2006;10:469-72.

17. Fillipl I, Driscoll JR, van Soolingen D, Kreiswirth BN, Kremer K, Valétudie G, et al. Global distribution of Mycobacterium tuberculosis spoligotypes. Emerg Infect Dis 2002;8:1347-9.

18. Brudey K, Driscoll JR, Rigouts L, Prodinger WM, Gori A, AlHajoj SA, et al. An appraisal of the geographic prevalence of major genotyping families of Mycobacterium tuberculosis complex through the updated SpolDB4 database. BMC Microbiol 2006;6:23. [CrossRef]

19. Bicmen C, Esen N, Graviss EA, Williams-Bouyer N, Ramaswamy SV, Yulug N. Molecular characterization of Mycobacterium tuberculosis isolates from Izmir, Turkey. New Microbiol 2007;30:229-40.

20. Wilson RW, Yang Z, Kelley M, Cave MD, Pogoda JM, Wallace $\mathrm{RJ} \mathrm{Jr}$, et al. Evidence from molecular fingerprinting of limited spread of drug-resistant tuberculosis in Texas. J Clin Microbiol 1999;37:3255-9. 